\title{
Calcineurin/NFAT signaling pathway mediates titanium particle-induced inflammation and osteoclast formation by inhibiting RANKL and M-CSF in vitro
}

\author{
YUNGE ZHANG* , PENG JIANG, WENBO LI, XIANGJIE LIU, YANG LU, ZHIPENG HUANG and KEGUAN SONG* \\ The Third Department of Orthopedics, The First Affiliated Hospital of Harbin Medical University, \\ Harbin, Heilongjiang 150001, P.R. China
}

Received December 29, 2016; Accepted August 17, 2017

DOI: $10.3892 / \mathrm{mmr} .2017 .7670$

\begin{abstract}
Wear particles serve a central role in periprosthetic osteolysis, which leads to the aseptic loosening of prostheses. In the present study a lentiviral vector was constructed to silence macrophage colony stimulating factor (M-CSF) and receptor activator of nuclear factor $\kappa-B$ ligand (RANKL) genes, which synergistically inhibit osteoclast formation and differentiation. To confirm the role of the calcineurin/nuclear factor of activated $\mathrm{T}$ cells (NFAT) pathway in osteolysis, we transduced murine macrophage/monocyte RAW264.7 cells with M-CSF-short hairpin (sh)RNA-RANKL-shRNA. Tumor necrosis factor- $\alpha$ $(\mathrm{TNF}-\alpha)$ protein levels were evaluated using enzyme-linked immunosorbent assay. Transduced RAW264.7 cells were cultured in Transwell chambers in the presence of $0.1 \mathrm{mg} / \mathrm{ml}$ titanium particles to investigate the capacity of TNF- $\alpha$ inhibition to reduce wear debris-induced inflammation. RANKL, M-CSF, TNF- $\alpha$, interleukin (IL)-1 $\beta$, IL-6 and NFATc1 mRNA levels were also assessed by reverse transcription-quantitative polymerase chain reaction. Osteoclastogenesis was measured by tartrate-resistant acid phosphatase (TRAP) mRNA quantification. Lentiviral-mediated double gene inhibition is known to be able to completely inhibit inflammatory osteolysis, simultaneously decreasing the number of NFATc1and TRAP-positive cells. The present study confirmed that the combined silencing of M-CSF and RANKL genes can inhibit the osteolysis induced by the wear particles around the prosthesis. The calcineurin/NFAT pathway serves a role in the prevention of prosthesis loosening.
\end{abstract}

Correspondence to: Professor Keguan Song, The Third Department of Orthopedics, The First Affiliated Hospital of Harbin Medical University, 23 Youzheng Street, Nangang, Harbin, Heilongjiang 150001, P.R. China

E-mail: songkeguan@sohhu.com

*Contributed equally

Key words: wear particle, osteoclastogenesis, calcineurin, nuclear factor of activated $\mathrm{T}$ cells, periprosthetic osteolysis

\section{Introduction}

For severe hip joint degeneration, post-traumatic and other types of end-stage osteoarthritis, the safest and most effective method of treatment is total hip arthroplasty (THA) (1). Since 1960, THA has become a cost-effective surgery to reduce joint pain and recover movement capabilities, which also makes it one of the most successful surgeries in the history of joint reconstruction (2). However, periprosthetic osteolysis remains a frequent complication following total hip replacement due to inflammatory responses to the microscopic particles from the bone-implant interface membrane $(3,4)$. Monocytes/macrophages, fibroblast and mesenchymal stem cells are major cell types and effector cells involved in inflammatory osteolysis. At the interface membrane of prostheses, macrophages phagocytose foreign particles into a larger cell containing inclusions forming the osteoclast precursor cells, which can also be isolated from a number of macrophages (5). Currently available evidence suggests that macrophage phagocytose wear particles by stimulating cellular responses, including the expansion of the cytoplasm and secretion of a variety of pro-inflammatory cytokines. These cells are capable of secreting a large number of inflammatory cytokines, including interleukin-1 (IL-1), tumor necrosis factor- $\alpha$ (TNF- $\alpha$ ) and IL-6 that stimulate osteoclast-induced bone resorption $(6,7)$. These inflammatory factors further increase the number of monocytes in the peripheral system and induce the transformation of macrophages into osteoclasts. The osteoclasts are terminal cells leads to osteolysis and bone resorption and are arthroplasty prosthesis loosening occurred the end effector cells and root cause. Osteoclasts cause osteolysis and bone resorption around the joint prosthesis, which is the main cause of joint prosthesis loosening.

The molecular mechanisms governing osteolysis and detailed mechanism of the formation of osteoclasts remain unknown. At present, the generally accepted process of osteolysis is that following stimulation of wear particles, they can activate a variety of cellular signaling pathways, regulate the expression of a number of signaling proteins and ultimately induce osteoclast differentiation and formation (8). Osteoclasts are large multinucleated cells characteristic of bone tissue, differentiated from hematopoietic stem cells 
and are involved in regulating physiological functions, bone homeostasis, and hematopoiesis. Osteoclasts differentiate under the control of two major cytokines, namely receptor activator of nuclear factor- $\kappa \mathrm{B}$ ligand (RANKL) and macrophage colony-stimulating factor (M-CSF) (9). Without these factors, osteoclast differentiation is delayed, inefficient and leads to early apoptosis (10). M-CSF binding to colony stimulating factor 1 receptor (c-fms) is a key signal for the proliferation and survival of osteoclast precursor cells. RANKL binding to RANK is a stimulating signal for osteoclast differentiation and bone resorption, and maturation of osteoclasts $(11,12)$. RANK interacts with RANKL when co-stimulatory signals including M-CSF are present. The encapsulated fraction of transmembrane receptor RANK first induces TNF receptor-associated factor (TRAF), which then activates multiple signal transduction pathways. Finally, through proto-oncogene c-Src, tartrate-resistant acid phosphatase (TRAP), cathepsin K, carbonic anhydrase 2 and a series of osteoclast-specific gene expression, osteoclast differentiation, function and apoptosis is regulated (13).

RANKL is a member of the TNF receptor ligand superfamily and is located in cells in a membrane-bound form with a soluble carboxyl terminal. Osteoblasts, bone marrow stromal cells and activated T lymphocytes express RANKL (14). RANK binds to RANKL, and is mainly expressed in monocytes/macrophages and in osteoclast precursor cells or on the surface of mature osteoclasts. RANK promotes osteoclast differentiation and activation, and prevents apoptosis of osteoclasts. Osteoblasts and bone marrow stromal cells also express another member of the tumor necrosis factor superfamily, osteoprotegerin (OPG). $\mathrm{OPG}$ is present in the form of soluble secretory proteins, which bind to RANK, thus competing with RANKL. Inhibiting the interaction between RANK and RANKL can lead to inhibition of osteoclast differentiation and function. Therefore, in the process of dynamic balance between bone remodeling and bone resorption, $\mathrm{RANKL/OPG}$ interaction is an important regulating mechanism $(15,16)$. RANKL binds to RANK and activates the downstream signaling pathway. RANKL lacks the enzyme active site and it binds to RANK through recruitment of linker molecules, including TRAF6, which in turn activates nuclear factor $\kappa \mathrm{B}(\mathrm{NF}-\kappa \mathrm{B})$, calcineurin/nuclear factor of activated $\mathrm{T}$ cells (CN/NFAT) and phosphatidylinositol signaling pathways.

M-CSF is a lineage specific cytokine located primarily in the bone marrow cavity. M-CSF is a ligand for colony stimulating factor 1 receptor membrane-bound form, and serves an important role in the proliferation, differentiation and maintenance activity of the mononuclear cells. Previous experiments (17) demonstrated that in the presence of bone resorption-stimulating factor, co-culturing of normal mouse skull bone marrow cells with other bone marrow cells generates osteoclasts. However, M-CSF-deficient mice failed to produce the osteoclast precursor cells and osteoclasts. The osteoclast formation potential was restored by adding exogenous M-CSF to M-CSF-deficient mice, which emphasizes the importance of these cytokines in osteoclast formation. Relevant studies have also demonstrated that M-CSF serves a critical role in the regulation of multiple steps involved in the regulation of human osteoclasts, including proliferation, differentiation and the fusion of their precursor cells $(18,19,20)$.

The role of CN/NFAT signaling pathways in bone metabolism, immunity and other systems has been extensively studied over previous years. Studies have indicated that the osteoclast differentiation begins with RANKL-mediated activation of the CN/NFAT signaling pathway. Upon this activation, transcription factor NFATc1 enters the nucleus to initiate osteoclast specific gene transcription $(21,22)$. NFATc1 is an essential transcription factor in the process of osteoclast differentiation and maturation as it regulates osteoclast specific gene expression (10). A previous study demonstrated that early in osteoclast differentiation, c-Fos is recruited to the NFATc1 promoter region and the interaction with RANKL signal induces the normal expression of NFATc1, which can promote osteoclast precursor cells to differentiate into mature osteoclasts (23). Gene chip studies have demonstrated that RANKL induces p50 and p65 subunit aggregation to the NFATc1 promoter region as a part of the $\mathrm{NF}-\kappa \mathrm{B}$ signaling pathway during osteoclast differentiation and maturation (24). The immunoreceptors on the surface of the cell membrane, including osteoclast-associated immunoglobulin-like receptor, pirin-A, surface triggering receptor expressed on myeloid cells 2 and signal regulatory protein- $\beta 1$, are essential for the reception of costimulatory signals during osteoclast differentiation and activation. Immunoreceptors, through their allosteric intracellular immunoreceptor tyrosine-based activation motif, detect changes in $\mathrm{Ca}^{2+}$ concentration and patina, activate $\mathrm{CN}$ and promote the expression of NFATc1, which induces osteoclast generation. In addition, LIM homeobox 2, interferon regulatory factor 8 , B-cell lymphoma 6 and other NFATc1 suppressor genes were significantly downregulated in osteoclast progenitor cells when RANKL was present, whereas RANKL gene-knockout mice demonstrated significantly increased expression of NFATc1 and osteoporosis $(25,26)$. Therefore, in the process of osteoclast differentiation and maturation, NFATc1 is a vital component of the signal transduction pathway leading to osteoclast formation. Previous studies have demonstrated that wear particles can enhance the activity of the CN/NFAT signaling pathway during osteoclast differentiation and promote osteoclast differentiation and function through this pathway $(27,28)$.

Wear particles can induce various types of inflammatory factors and periprosthetic macrophages, which, via a series of biological reactions, induce osteolysis (29). A previous study from the United States indicates that the proportion of metal interfaces in joint prostheses remains high (30), and therefore the incidence of osteolysis induced by metal wear particles remains relatively high. Therefore, titanium particles were chosen as the research subject of this paper. The authors previously demonstrated that lentiviruses can be successfully used to induce the release of inflammatory cytokines around a prosthesis (31). In the present study a lentiviral vector containing M-CSF-shRNA and RANKL-shRNA was constructed to inhibit the upstream regulatory factors of osteoclast formation, further proving the key role of NFATc1 in the CN/NFAT signaling pathway. The aim of the present study was to identify a target for the inhibition of osteolysis around the prosthesis. 


\section{Materials and methods}

Cell culture. Murine osteoclast progenitor macrophage/monocyte RAW264.7 cells (American Type Culture Collection, Manassas, VA, USA) suitable for host transfection were used. Cells were cultured in $\alpha$-minimum essential medium ( $\alpha$-MEM; Hyclone; GE Healthcare Life Sciences, Logan, UT, USA) containing $10 \%$ fetal bovine serum (FBS; Hyclone; GE Healthcare Life Sciences), $100 \mathrm{U} / \mathrm{ml}$ penicillin (Gibco; Thermo Fisher Scientific, Inc., Waltham, MA, USA) and $100 \mathrm{~g} / \mathrm{ml}$ streptomycin (Gibco; Thermo Fisher Scientific, Inc.) at $37.6^{\circ} \mathrm{C}$ under $5 \% \mathrm{CO}_{2}$ and $95 \%$ humidity. Cells at the prosthesis interface were plated with $\alpha$-MEM containing FBS and treated or untreated with M-CSF-shRNA-RANKL-shRNA depending on the experimental design. Cells were cultured in differentiation medium with or without the Ti particles $(0.1 \mathrm{mg} / \mathrm{ml})$. The RAW264.7 cultures were divided into three groups: i) Ti particles only (Ti group); ii) Ti particles and lentiviral vector (lenti) -M-CSF-RANKL (lenti-M-CSF-RANKL group); and iii) neither wear particles nor lenti-M-CSF-RANKL (control group).

Lentiviral vector construction and recombinant lentivirus production. The shRNA expression vector PLV-shRNA-green fluoresecent protein (GFP; Shanghai GeneChem Co., Ltd., Shanghai, China) was used in the present study. This vector contained two U6 promoters, which were independent of each other, so that two interfering genes could be activated at the same time and the interference efficiency was independent of each other. The RANKL interference sequence was cloned into the PLV-shRNA-GFP vector by restriction endonucleases HpaI and XhoI (Shanghai GeneChem Co., Ltd.) to form a lentiviral vector PLV-shRNA-GFP (RANKL-shRNA). The U6-shRNA expression cassette was cloned into the lentiviral vector PLV-shRNA-GFP (RANKL-shRNA) using NotI and NsiI (Shanghai GeneChem Co., Ltd.) sites. The interference sequence for the second gene was cloned into the lentiviral vector using the restriction endonuclease sites of $\mathrm{HpaI}$ and XhoI to construct a double RNA vector PLV-M-CSF-shRNA -RANKL-shRNA-GFP. This construct was transfected into cells to induce interference with M-CSF and RANKL genes simultaneously. Following construction, the recombined lentiviral vector and pPACK Packaging Plasmid mix (Invitrogen; Thermo Fisher Scientific, Inc.) were cotransfected into 293T cells (GeneChem, Inc., Daejeon, Korea). The pGag/Pol, pRev and pVSV-G packaging vectors, and recombinant lentivirus were packaged into plasmid vectors, and the recombinant lentivirus was amplified by transforming 293T cells with the packaging plasmids $(0.5 \mu \mathrm{g} / \mu \mathrm{l})$ using Lipofectamine ${ }^{\mathrm{TM}} 2000$ (Invitrogen; Thermo Fisher Scientific, Inc.). At $48 \mathrm{~h}$ post-transfection, the lentiviruses were harvested and centrifuged at $4,000 \mathrm{x}$ g for $10 \mathrm{~min}$ at $4^{\circ} \mathrm{C}$ to remove cell debris. Condensation was performed by filtration of the supernatant into a filtrate collection tube through a filter cup, followed by centrifugation at $4,000 \mathrm{x} \mathrm{g}$ for $13 \mathrm{~min}$ at $4^{\circ} \mathrm{C}$. The filter cup was removed, a sample collection cup was inserted into the filtrate collection tube and the solution was centrifuged at $1,000 \mathrm{x} \mathrm{g}$ for $2 \mathrm{~min}$ at $4^{\circ} \mathrm{C}$. Finally, a concentrated lentivirus solution was obtained, with a final titer of $1.5 \times 10^{9}$ transducing units/l.
Titanium particle preparation. In the present study, commercial pure titanium particles were obtained from Johnson Matthey Pharma Services (Ward Hill, MA, USA) and the average particle diameter was $4.5 \mathrm{~mm}$ (range, $1-10 \mathrm{~mm}$ ). The titanium particles were dissolved in $75 \%$ ethanol, washed in PBS (Wuhan Boster Biological Technology, Ltd., Wuhan, China) at room temperature 4 times, $1 \mathrm{~h} /$ wash, and then washed in PBS (Wuhan Boster Biological Technology, Ltd., Wuhan, China) and incubated in $100 \%$ ethanol overnight. Finally, the treated titanium particles were soaked in $4^{\circ} \mathrm{C} \alpha$-MEM for storage. Titanium particles used in the experiment had endotoxin levels $<0.2$ endotoxin units/ml verified using a Limulus Amebocyte Lysate assay (Xiamen Houshiji, Ltd., Xiamen, China), according to the manufacturer's protocol. It is reported from the literature that these particles are similar to particles produced in the surrounding tissue of the prosthesis (32).

MTT assay. The cytotoxicity of Ti particles in RAW264.7 cells transfected with M-CSF-shRNA-RANKL-shRNA was examined using the MTT assay. The cells $\left(5 \times 10^{3}\right.$ cells/well) were cultured in 96-well tissue culture plates for $24 \mathrm{~h}$ and incubated with $0.5 \mathrm{mg} / \mathrm{ml} \mathrm{MTT}$ at $37^{\circ} \mathrm{C}$ for $4 \mathrm{~h}$. Following the removal of the supernatant, the insoluble formazan crystals were dissolved in $200 \mu \mathrm{l}$ dimethyl sulfoxide and the absorbance was measured using a Synergy HT microtiter plate reader (BioTek Instruments, Inc., Winooski, Vermont, USA) at a wavelength of $570 \mathrm{~nm}$.

TRAP staining of osteoclasts. TRAP osteoclast-specific staining can be used to observe the formation of osteoclasts. A total of three osteoclast specimens were taken for analysis from an osteolytic area. After 7 days RAW264.7 cells were fixed with $4 \%$ paraformaldehyde (at $37^{\circ} \mathrm{C}$ for $20 \mathrm{~min}$ ) and stained at $37^{\circ} \mathrm{C}$ for $4 \mathrm{~h}$ for TRAP using a leukocyte acid phosphatase staining kit (Sigma-Aldrich; Merck KGaA, Darmstadt, Germany) according to the manufacturer's protocol, for the analysis of the formation of osteoclasts. TRAP-positive cells with $>3$ nuclei were considered differentiated osteoclast-like cells (33).

$R N A$ isolation and reverse transcription-quantitative polymerase chain reaction $(R T-q P C R)$. RT-qPCR was used to assess the expression of RANKL, M-CSF, NFATc1, TNF- $\alpha$, IL-1 $\beta$ and IL-6 in RAW264.7 cells under different conditions. Total RNA was extracted from RAW264.7 cultures using TRIzol (Invitrogen; Thermo Fisher Scientific, Inc.), according to the manufacturer's protocol. RNA purity was determined using the 260/280 $\mathrm{nm}$ absorbance ratio (NanoDrop; Thermo Fisher Scientific, Inc., Wilmington, DE, USA). Each first-strand cDNA was synthesized with $2 \mathrm{mg}$ total RNA using the TaqMan Real-Time PCR Master Mixes (Fermentas; Thermo Fisher Scientific, Inc., Pittsburgh, PA, USA) and 1/10th of the total cDNA was used for each PCR mixture containing EXPRESS SYBR-Green (Takara Bio, Inc., Otsu, Japan) and PCR Supermix (Fermentas; Thermo Fisher Scientific, Inc.). The primers and thermal cycling conditions for RANKL, M-CSF, TNF- $\alpha$, IL-1 $\beta$, IL- 6 and NFATc1 are listed in Table I. The cycling conditions were: $95^{\circ} \mathrm{C}$ for $15 \mathrm{sec}, 95^{\circ} \mathrm{C}$ for $5 \mathrm{sec}$ and $60^{\circ} \mathrm{C}$ for $30 \mathrm{sec}$. A total of 45 cycles were run. Relative mRNA expression of selected 
Table I. Primers for the reverse transcription-quantitative polymerase chain reaction.

\begin{tabular}{lll}
\hline Target & \multicolumn{1}{c}{ Forward primer $\left(5^{\prime}-3^{\prime}\right)$} & \multicolumn{1}{c}{ Reverse primer (3'-5') } \\
\hline TNF- $\alpha$ & TCCTCACCCACACCGTCAG & GCTGAGTTGGTCCCCCTTC \\
IL-1 $\beta$ & TTCTCGCAGCAGCACATC & CAGCAGGTTATCATCATCATCC \\
IL-6 & TCCATCCAGTTGCCTTCTTG & TTTCTCATTTCCACGATTTCCC \\
NFATc1 & CAACGCCCTGACCACCGATAG & GGCTGCCTTCCGTCTCATAGT \\
RANKL & AGATTTGCAGGACTCGACTC & CCCACA ATGTGTTGCAGTTC \\
M-CSF & CCACTTGTAGAACAGGAGGCCC & GCTTGAGGGCAAGAGAAGTACC \\
GAPDH & TGGTGAAGGTCGGTGTGAAC & GCTCCTGGAAGATGGTGATGG
\end{tabular}

TNF- $\alpha$, tumor necrosis factor- $\alpha$; IL, interleukin; NFATc1, activated T cell nuclear factor $\mathrm{c} 1$; RANKL, receptor activator of nuclear factor $\kappa$-B ligand; M-CSF, macrophage colony-stimulating factor.

genes was normalized to the GAPDH reference gene and quantified using the $2^{-\Delta \Delta \mathrm{Cq}}$ method (34).

ELISA. Following $48 \mathrm{~h}$ of incubation with recombinant lentivirus (produced as described above), the RAW264.7 cells were incubated with or without Ti particles in the presence or absence of M-CSF-shRNA-RANKL-shRNA for $24 \mathrm{~h}$ and the cell supernatants were collected and centrifuged at $1,200 \mathrm{x} \mathrm{g}$ for $8 \mathrm{~min}$ at room temperature to remove the cell particles. Aliquots were stored at $-20^{\circ} \mathrm{C}$ for TNF- $\alpha$ measurement. Mouse-specific ELISA kits were used to analyze the amount of TNF- $\alpha$. ELISA kit (Mouse TNF- $\alpha$ Tumor Necrosis Factor- $\alpha$ ELISA kit; cat. no. E-EL-M0049; R\&D Systems, Inc., Minneapolis, MN, USA) was used for quantitative measurement, according to the manufacturer's protocol.

Statistical analysis. All data were expressed as the mean \pm standard deviation of the mean. Differences between groups were analyzed using one-way analysis of variance followed by Dunnett's test. $\mathrm{P}<0.05$ was considered to indicate a statistically significant difference. Statistical analyses were performed using SPSS software (version 17.0; SPSS, Inc., Chicago, IL, USA).

\section{Results}

Effects of Ti particles and lenti-M-CSF-RANKL on cell viability. In the presence of titanium particles, RAW264.7 cells cultured for 24-72 $\mathrm{h}$ and analyzed using the MTT assay, revealed no significant differences between the cells transfected with lenti-M-CSF-RANKL and the untransfected cells (Fig. 1). Therefore, it was observed that the treatments had no significant effects on the activity of the cells in Ti group and Lenti-M-CSF-RANKL group.

Effect of lenti-M-CSF-RANKL on Ti particle-induced expression of inflammatory factors in RAW264.7 cells. The murine macrophage/monocyte cell line RAW264.7 was treated with Ti or lenti-M-CSF-RANKL, or left untreated. Transduced RAW264.7 cells were cultured in the presence of $0.1 \mathrm{mg} / \mathrm{ml}$ Ti particles to investigate the capacity of M-CSF and RANKL mediated inhibition to reduce wear debris-induced inflammation. To evaluate the particle-induced inflammatory

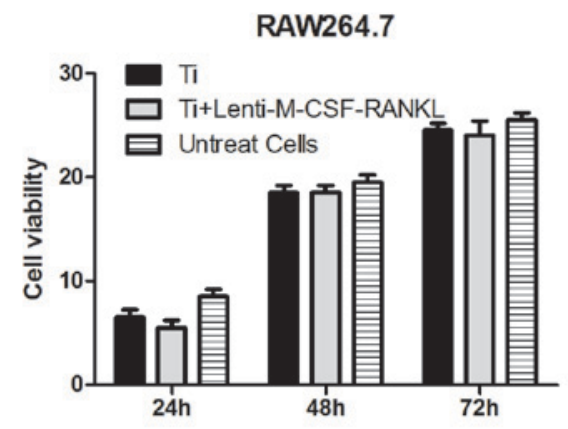

Figure 1. Effects of lenti-M-CSF-RANKL on cell viability, determined using an MTT assay. RAW264.7 were treated with Ti and Lenti-M-CSF-RANKL RAW264.7 for 24, 48 and $72 \mathrm{~h}$. Data are presented as the mean \pm standard deviation. M-CSF, macrophage colony-stimulating factor; lenti, lentiviral vector; RANKL, receptor activator of nuclear factor $\kappa-\mathrm{B}$ ligand.

response, the present study examined the expression levels of proinflammatory cytokines, including TNF- $\alpha$, IL-1 $\beta$ and IL-6 (Fig. 2). Following a $24 \mathrm{~h}$ incubation with a combination of Ti particles and lenti-M-CSF-RANKL, the TNF- $\alpha$ expression was inhibited in the RAW264.7 cells but not in the Ti group cells, as determined using ELISA analysis (Fig. 2B). The mRNA levels of TNF- $\alpha$, IL- $1 \beta$ and IL- 6 were also assessed using RT-qPCR (Fig. 2A, C and D).

Following $24 \mathrm{~h}$ of culture, the concentration of TNF- $\alpha$, IL-1 $\beta$ and IL- 6 was significantly increased in the cell lysate of cultures containing wear particles compared with those without the particles (Fig. 2A, C and D). Contrastingly (from the experimental results), TNF- $\alpha, \mathrm{L}-1 \beta$ and IL-6 mRNA levels were significantly decreased in cultures containing RAW264.7 cells infected with lenti-M-CSF-RANKL compared with the Ti group (Fig. 2; all P<0.05). Downregulation of M-CSF and RANKL resulted in a decrease in the mRNA expression levels of IL-1 $\beta$ (Fig. 2C) and IL-6 (Fig. 2D), compared with the Ti group, which indicates that M-CSF controls the mRNA expression levels of IL-6 and IL-1 $\beta$.

Inhibition of osteoclastogenesis by transduction of RAW264.7 cells with lenti-M-CSF-RANKL. To determine the effect of each treatment on the stimulation of osteoclastogenesis, TRAP-positive multinucleated RAW264.7 cells (osteoclasts) 

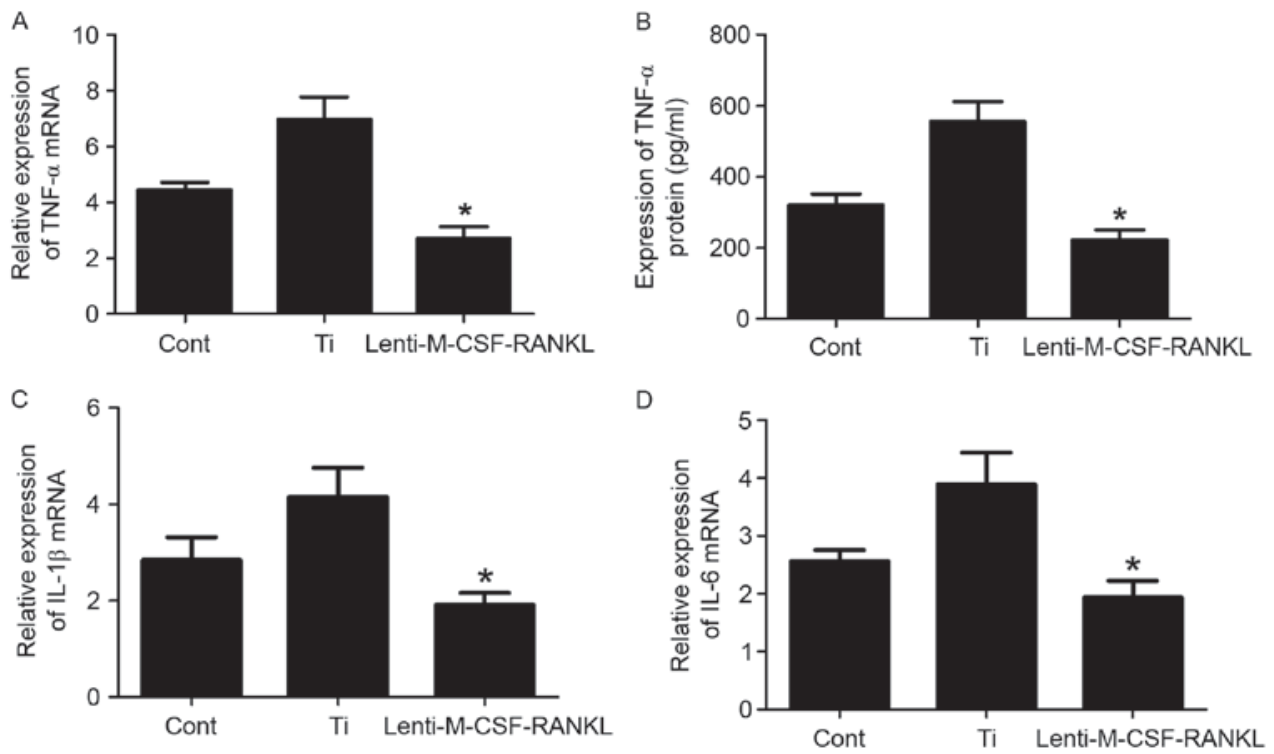

Figure 2. mRNA and protein expression levels of proinflammatory cytokines in RAW264.7 cells. RAW264.7 cells were treated with or without Ti particles in the presence or absence of Lenti-M-CSF-RANKL for $48 \mathrm{~h}$. (A) mRNA and (B) Protein levels of TNF- $\alpha$ were assessed using an ELISA following exposure to Ti particles, or Ti particles and Lenti-M-CSF-RANKL for $24 \mathrm{~h}$. mRNA expression levels of (C) IL-1 $\beta$ and (D) IL-6 were downregulated by lenti-M-CSF-RANKL, compared with the Ti group. Data are presented as the mean \pm standard deviation. "P<0.05 vs. the Ti group. M-CSF, macrophage colony-stimulating factor; lenti, lentiviral vector; RANKL, receptor activator of nuclear factor $\kappa-B$ ligand; IL, interleukin; TNF- $\alpha$, tumor necrosis factor- $\alpha$.

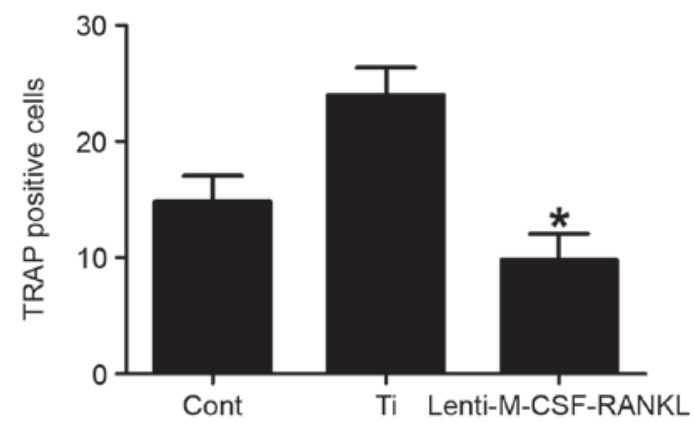

Figure 3. Inhibition of M-CSF and RANKL by transduction of lenti-M-CSF-RANKL. The number of TRAP-positive cells was decreased in groups treated with lenti-M-CSF-RANKL in comparison with Ti alone. Data are presented as the mean \pm standard deviation. ${ }^{*} \mathrm{P}<0.05$ vs. the $\mathrm{Ti}$ group. M-CSF, macrophage colony-stimulating factor; lenti, lentiviral vector; RANKL, receptor activator of nuclear factor $\kappa-B$ ligand; TRAP, tartrate-resistant acid phosphatase.

were examined. A total of 7 days post Ti-particle or lenti-M-CSF-RANKL stimulation, RAW264.7 cells were fixed and stained for TRAP. The number of TRAP positive cells was significantly decreased $(\mathrm{P}<0.05)$ in cultures infected with lenti-M-CSF-RANKL compared with the Ti only group (Fig. 3). This data therefore suggests that the lentiviral treatment was able to inhibit the Ti particle-induced osteoclastogenesis.

Expression of NFATc1 following treatment with Ti particles and lenti-M-CSF-RANKL. A previous study demonstrated that upon inhibition of RANK activity, osteoclast function in bone resorption decreases significantly (25). In the present study, the levels of NFATc1 mRNA in RAW264.7 cells when cultured in with or without $\mathrm{Ti}$ particles or lenti-M-CSF-RANKL were determined. At $48 \mathrm{~h}$ post transduction, in the lenti-M-CSF-RANKL group, the expression of

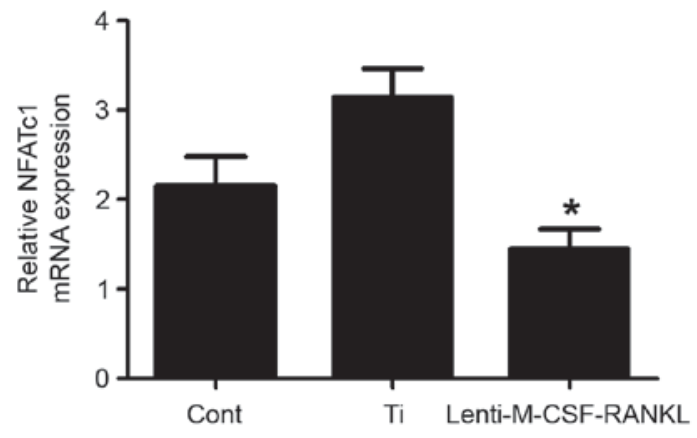

Figure 4. Relative mRNA expression of NFATc1 in RAW264.7 cells. Data are presented as the mean \pm standard deviation. ${ }^{*} \mathrm{P}<0.05$ vs. the Ti group. M-CSF, macrophage colony-stimulating factor; lenti, lentiviral vector; RANKL, receptor activator of nuclear factor $\kappa-B$ ligand; NFAT, nuclear factor of activated T cells.

NFATc1 in RAW264.7 cells was reduced (Fig. 4). The levels of RANKL and M-CSF mRNA were also analyzed prior to and following transfection. The results demonstrated that the expression of RANKL and M-CSF was significantly decreased following lentiviral transfection (both $\mathrm{P}<0.05$; Figs. 5 and 6), indicating that the lentivirus was effective. The results suggest that the expression of NFATc1 positively correlates with the formation of osteoclasts.

\section{Discussion}

At present, aseptic loosening of joint replacement prostheses is the most prevalent reason for joint revision surgeries (35). Due to the increasing median age of the population and a rise in the use of prosthetics at a younger age, the number of joint replacement procedures is increasing and the number of joint renovations is also predicted to continue to rise in the future (36). At present, the best strategy for the treatment of 
Raw264.7 Cell

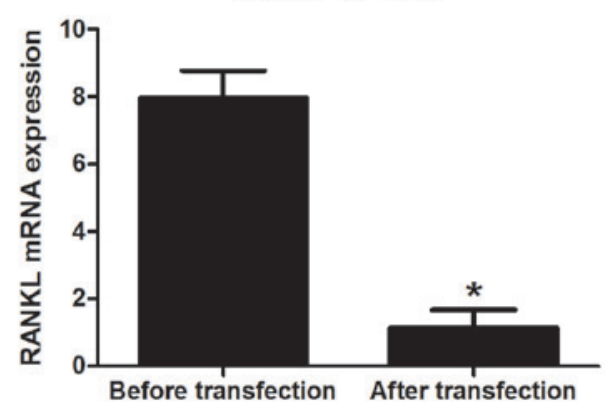

Figure 5. RANKL mRNA levels following transfection with the macrophage colony-stimulating factor-RANKL lentiviral vector. Data are presented as the mean \pm standard deviation. ${ }^{*} \mathrm{P}<0.05$ vs. the untransfected group. RANKL, receptor activator of nuclear factor $\mathrm{\kappa}-\mathrm{B}$ ligand.

aseptic loosening is joint revision. Therefore, investigation of the process of pathogenesis of aseptic loosening of the prosthesis, and seeking effective prevention and treatment measures can prolong the durability of the prosthesis. This project studied the formation and differentiation of osteoclasts with an emphasis on two critical factors: M-CSF and RANKL. The use of gene silencing technology allowed for synergistic inhibition of periprosthetical osteolysis induced by wear particles. The present study investigated the role of CN/NFAT signaling pathway and RANKL receptor in the differentiation of osteoclasts. Inhibition of M-CSF and RANKL allowed for determination of the molecular mechanisms underlying osteoclast differentiation and maturation. The gathered evidence provides experimental basis for the treatment of periprosthetic osteolysis induced by wear particles.

In the present study, a lentiviral vector containing M-CSF-shRNA and RANKL-shRNA double sequences was constructed, which can effectively inhibit the inflammatory reaction and osteoclast formation, and confirmed the role of these genes in the wear particle-induced osteolysis around the prostheses. The levels of RANKL and M-CSF mRNA were also analyzed prior to and following transfection. The results demonstrated that the expression of RANKL and M-CSF was significantly decreased following lentiviral transfection, indicating that the lentivirus was successfully constructed and met the requirements of the study. Cells around the prosthesis (osteoclasts, macrophages, fibroblasts and osteoblasts) engulfed the wear particles, which could not have been otherwise degraded by the bodily enzymes. Wear particles stimulate the release of IL-1, IL-6, TNF- $\alpha$, putative prostaglandin E2 synthase, OPG, RANKL, M-CSF, vascular endothelial growth factor $\mathrm{A}$ and other chemokines, leading to decreased osteoblast activity, increased osteoclast differentiation, cell damage, cell apoptosis and necrosis. As a result, the particles are re-released into the extracellular matrix to become repeatedly phagocytosed leading to repeated activation of phagocytes which secrete more inflammatory cytokines and protease enzymes, to induce, maintain and exacerbate wear particles induced chronic inflammatory and apoptotic responses. This effectively leads to a decrease in the number of osteoblasts and increased osteoclast differentiation (18).
Raw264.7 Cell

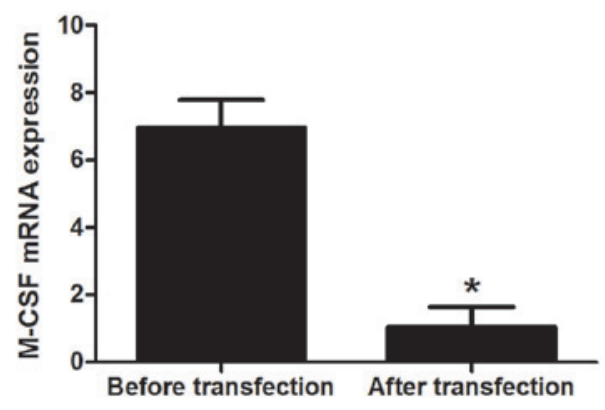

Figure 6. M-CSF mRNA levels following transfection with the macrophage M-CSF-receptor activator of nuclear factor $\kappa$-B ligand lentiviral vector. Data are presented as the mean \pm standard deviation. ${ }^{*} \mathrm{P}<0.05$ vs. the untransfected group. M-CSF, macrophage colony-stimulating factor.

$\mathrm{M}-\mathrm{CSF}$ and NF- $\mathrm{KB}$ are involved in the differentiation of osteoclasts, which is the only multinucleated cell derived from monocyte-macrophage precursors with bone resorption activity. The binding of M-CSF and RANKL to cell surface receptors provides signals for osteoclast survival and proliferation. The binding activates the corresponding signal transduction pathways allowing differentiating osteoclasts to express specific genes to eventually form mature osteoclasts and perform bone resorption functions (37). It has been observed that various cytokines (M-CSF, RANKL, TNF- $\alpha$ and IL-1) that mediate osteoclast differentiation and maturation, can directly and indirectly regulate the expression of key nuclear genes through complex signaling pathways, to promote or inhibit the differentiation of osteoclasts. NF- $\mathrm{kB}$-associated activator protein 1 (AP-1) transcription factors, mainly c-Fos, c-Jun and NFAT $(13,16,19)$ induce transcription of TRAP, calcitonin receptor, histone $\mathrm{K}$, carbonylase II and other genes promoting osteoclast differentiation. Multiple signaling pathways interact forming a complex regulatory network. TRAF6 is an essential upstream effector in RANKL signaling pathway, Inhibitor of $\kappa \mathrm{B}$ kinase activates NF- $\mathrm{KB}$ causing disassociation of the inhibitor of $\kappa \mathrm{B}$ subunit and rapid translocation of the transcription factor into the nucleus. The transcription factor interacts with promoter regions of a series of genes, including IL-1, IL-6, TNF- $\alpha$ and M-CSF, to initiate their transcription. However, the inflammatory cytokines can also activate osteoclast differentiation $(20,38)$.

Research has demonstrated that TNF- $\alpha$ is positively regulated in osteoblasts by expression of RANKL, M-CSF and IL-6, and enhances the activity of osteoclast precursor cells to promote osteoclast differentiation. IL-1 is induced by M-CSF and IL-6 production, and is expressed in a paracrine manner to activate jun $\mathrm{N}$-terminal kinase (JNK) and promote osteoclast precursor cell differentiation into mature osteoclasts. In addition, TNF- $\alpha$, IL-1 and IL- 6 can also activate the NF- $\mathrm{KB}$ signaling pathway $(39,40)$ causing secretion of more inflammatory factors, reinforcing the inflammatory response. Sabokbar et al (41) also observed that inhibiting RANKL-induced formation of osteoclasts was more evident in the presence of a small amount of wear particles but when a large number of wear particles accumulated around the prosthesis, cytokines M-CSF and TNF- $\alpha$ were sufficient to induce 
osteoclast differentiation. TNF- $\alpha$ and IL-1 can also promote synergistic osteolysis, suggesting that when numerous wear particles accumulate around the prosthesis, M-CSF and other inflammatory cytokines may also also represent a reasonable system to directly induce osteolysis. The results of the present study demonstrated that the inhibition of M-CSF and RANKL perturbs osteoclast formation and leads to osteolysis, which supports the results obtained by Sabokbar et al (41). Inhibiting M-CSF and RANKL can completely inhibit osteoclastogenesis and the release of TNF- $\alpha$. These results provided grounds for the gene therapy of chronic osteolytic diseases. The above experiment confirmed that titanium particles can induce osteoclastogenesis and promote the release of inflammatory factors.

NFAT is a $\mathrm{Ca}^{2+}$ regulatory transcription factor family including five members, NFATc1-4 and NFAT5, which have been described in osteoclasts. NFATcl is a key target gene downstream of the RANK signal and is a fundamental effector molecule responsible for osteoclast differentiation. Previous studies demonstrated that RANKL could activate TRAF6/NF- $\kappa$ B, JNK/AP-1 and $\mathrm{Ca}^{2+} / \mathrm{CN}$ pathways and activate NFATc1 to induce differentiation of osteoclast $(13,16)$. Contrastingly, specific inhibitor $\mathrm{CN}$ can inhibit osteoclast differentiation. In osteoclast progenitor Fos double-negative cells, the expression level of NFATc1 decreased in the presence of RANKL. NFATc1 gene silencing can effectively inhibit wear particle-induced osteoclast differentiation (24), indicating that NFATc1 is indispensable in the activation of osteoclasts. As NFATc1 is induced by RANKL and regulates osteoclast differentiation $(18,19)$, wear particles promote osteoclast differentiation and inhibit osteoblast differentiation and function by activating CN/NFAT signaling pathway $(24,25)$. The present study demonstrated that the expression of NFATc1 in the presence of titanium particles is significantly increased, but it decreases significantly in the lentiviral group, indicating that the titanium particle-induced activation of the NFAT pathway is implicated in the osteoclastogenesis process.

In conclusion, the present study confirmed that the technology used is reasonable and effective, and may provide a novel effective way to prevent and treat the osteoclast osteolysis induced by the abrasive particles. At the same time, inhibition of wear particle-induced osteolysis around the prosthesis by gene silencing, can contribute to gene-associated drug research and development.

\section{Acknowledgements}

All experiments were completed in the Laboratory of Cardiology of The First Affiliated Hospital of Harbin Medical University (Harbin, China). The authors of the present study would like to thank the managers and staff for their hospitality, time and opinions. The present study was supported by funding from the National Natural Science Foundation of China (grant no. 81270635).

\section{References}

1. Gallo J, Goodman SB, Konttinen YT and Raska M: Particle disease: Biologic mechanisms of periprosthetic osteolysis in total hip arthroplasty. Innate Immun 19: 213-224, 2013.

2. Mellon SJ, Liddle AD and Pandit H: Hip replacement: Landmark surgery in modern medical history. Maturitas 75: 221-226, 2013.
3. Abu-Amer Y, Darwech I and Clohisy JC: Aseptic loosening of total joint replacements: Mechanisms underlying osteolysis and potential therapies. Arthritis Res Ther 9 (Suppl 1): S6, 2007.

4. Jacobs JJ, Hallab NJ, Urban RM and Wimmer MA: Wear particles. J Bone Joint Surg 88 (Supple 2): S99-S102, 2006.

5. Ollivere B, Wimhurst JA, Clark IM and Donell ST: Current concepts in osteolysis. J Bone Joint Surg Br 94: 10-15, 2012.

6. Yamanaka Y, Clohisy JC, Ito H, Matsuno T and Abu-Amer Y: Blockade of JNK and NFAT pathways attenuates orthopedic particle-stimulated osteoclastogenesis of human osteoclast precursors and murine calvarial osteolysis. J Orthop Res 31: 67-72, 2013.

7. Jones LC, Frondoza C and Hungerford DS: Immunohistochemical evaluation of interface membranes from failed cemented and uncemented acetabular components. J Biomed Mater Res 48: 889-898, 1999.

8. Rao AJ, Gibon E, Ma T, Yao Z, Smith RL and Goodman SB: Revision joint replacement, wear particles, and macrophage polarization. Acta Biomater 8: 2815-2823, 2012.

9. Teitelbaum SL and Ross FP: Genetic regulation of osteoclast development and function. Nat Rev Genet 4: 638-649, 2003.

10. Ikeda $\mathrm{K}$ and Takeshita $\mathrm{S}$ : The role of osteoclast differentiation and function in skeletal homeostasis. J Biochem 159: 1-8, 2016.

11. Suda T, Takahashi N, Udagawa N, Jimi E, Gillespie MT and Martin TJ: Modulation of osteoclast differentiation and function by the new members of the tumor necrosis factor receptor and ligand families. Endocr Rev 20: 345-357, 1999.

12. Lacey DL, Timms E, Tan HL, Kelley MJ, Dunstan CR, Burgess T, Elliott R, Colombero A, Elliott G, Scully S, et al: Osteoprotegerin ligand is a cytokine that regulates osteoclast differentiation and activation. Cell 93: 165-176, 1998.

13. Boyle WJ, Simonet WS and Lacey DL: Osteoclast differentiation and activation. Nature 423: 337-342, 2003.

14. Kim JH and Kim N: Signaling pathways in osteoclast differentiation. Chonnam Med J 52: 12-17, 2016.

15. Tanaka H, Mine T, Ogasa H, Taguchi T and Liang CT: Expression of RANKL/OPG during bone remodeling in vivo. Biochem Biophys Res Commun 411: 690-694, 2011.

16. Nakashima $\mathrm{T}$ and Takayanagi $\mathrm{H}$ : New regulation mechanisms of osteoclast differentiation. Ann N Y Acad Sci 1240: E13-E18, 2011.

17. Tsurukai T, Udagawa N, Matsuzaki K, Takahashi N and Suda T: Roles of macrophage-colony stimulating factor and osteoclast differentiation factor in osteoclastogenesis. J Bone Miner Metab 18: 177-184, 2000.

18. Jiang Y, Jia T, Gong W, Wooley PH and Yang SY: Titanium particle-challenged osteoblasts promote osteoclastogenesis and osteolysis in a murine model of periprosthestic osteolysis. Acta Biomater 9: 7564-7572, 2013.

19. Takayanagi H: Osteoclast differentiation and activation. Clin Calcium 17: 484-492, 2007.

20. Yamashita T, Takahashi N and Udagawa N: New roles of osteoblasts involved in osteoclast differentiation. World J Orthop 3: $175-181,2012$.

21. Negishi-Koga $\mathrm{T}$ and Takayanagi $\mathrm{H}$ : Ca2+-NFATc1 signaling is an essential axis of osteoclast differentiation. Immunol Rev 231: 241-256, 2009.

22. Aliprantis AO, Ueki Y, Sulyanto R, Park A, Sigrist KS, Sharma SM, Ostrowski MC, Olsen BR and Glimcher LH: NFATc1 in mice represses osteoprotegerin during osteoclastogenesis and dissociates systemic osteopenia from inflammation in cherubism. J Clin Invest 118: 3775-3789, 2008.

23. Takayanagi H, Kim S, Koga T, Nishina H, Isshiki M, Yoshida H, Saiura A, Isobe M, Yokochi T, Inoue J, et al: Induction and activation of the transcription factor NFATc1 (NFAT2) integrate RANKL signaling in terminal differentiation of osteoclasts. Dev Cell 3: 889-901, 2002.

24. Asagiri M, Sato K, Usami T, Ochi S, Nishina H, Yoshida H, Morita I, Wagner EF, Mak TW, Serfling E and Takayanagi H: Autoamplification of NFATc1 expression determines its essential role in bone homeostasis. J Exp Med 202: 1261-1269, 2005.

25. Kim JH, Youn BU, Kim K, Moon JB, Lee J, Nam KI, Park YW, O'Leary DD, Kim KK and Kim N: Lhx2 regulates bone remodeling in mice by modulating RANKL signaling in osteoclasts. Cell Death Differ 21: 1613-1621, 2014.

26. Zhao B, Takami M, Yamada A, Wang X, Koga T, Hu X, Tamura T, Ozato K, Choi Y, Ivashkiv LB, et al: Interferon regulatory factor- 8 regulates bone metabolism by suppressing osteoclastogenesis. Nat Med 15: 1066-1071, 2009. 
27. Liu F, Zhu Z, Mao Y, Liu M, Tang T and Qiu S: Inhibition of titanium particle-induced osteoclastogenesis through inactivation of NFATc1 by VIVIT peptide. Biomaterials 30: 1756-1762, 2009.

28. Maoqiang L, Zhenan Z, Fengxiang L, Gang W, Yuanqing M, Ming L, Xin Z and Tingting T: Enhancement of osteoblast differentiation that is inhibited by titanium particles through inactivation of NFATc1 by VIVIT peptide. J Biomed Mater Res A 95: 727-734, 2010.

29. Howie DW, Neale SD, Haynes DR, Holubowycz OT, McGee MA, Solomon LB, Callary SA, Atkins GJ and Findlay DM: Periprosthetic osteolysis after total hip replacement: Molecular pathology and clinical management. Inflammopharmacology 21: 389-396, 2013.

30. Lehil MS and Bozic KJ: Trends in total hip arthroplasty implant utilization in the United States. J Arthroplasty 29: 1915-1918, 2014.

31. Peng L, Wang H, Song K, Wang H and Liu P: Lentivirus-mediated TNF- $\alpha$ gene silencing and overexpression of osteoprotegerin inhibit titanium particle-induced inflammatory response and osteoclastogenesis in vitro. Mol Med Rep 13: 1010-1018, 2016.

32. Clohisy JC, Hirayama T, Frazier E, Han SK and Abu-Amer Y: NF-kB signaling blockade abolishes implant particle-induced osteoclastogenesis. J Orthop Res 22: 13-20, 2004.

33. Zheng H, Yu X, Collin-Osdoby $\mathrm{P}$ and Osdoby P: RANKL stimulates inducible nitric-oxide synthase expression and nitric oxide production in developing osteoclasts. An autocrine negative feedback mechanism triggered by RANKL-induced interferon-beta via NF-kappaB that restrains osteoclastogenesis and bone resorption. J Biol Chem 281: 15809-15820, 2006.
34. Livak KJ and Schmittgen TD: Analysis of relative gene expression data using real-time quantitative PCR and the 2(-Delta Delta C(T)) method. Methods 25: 402-408, 2001.

35. Del Buono A, Denaro V and Maffulli N: Genetic susceptibility to aseptic loosening following total hip arthroplasty: A systematic review. Br Med Bull 101: 39-55, 2012.

36. Singh JA and Sloan JA: Health-related quality of life in veterans with prevalent total knee arthroplasty and total hip arthroplasty. Rheumatology (Oxford) 47: 1826-1831, 2008.

37. Kim JH and Kim N: Regulation of NFATc1 in Osteoclast Differentiation. J Bone Metab 21: 233-241, 2014.

38. Zaidi M, Blair HC, Moonga BS, Abe E and Huang CL: Osteoclastogenesis, bone resorption, and osteoclast-based therapeutics. J Bone Miner Res 18: 599-609, 2003.

39. Zhang K and Kaufman RJ: From endoplasmic-reticulum stress to the inflammatory response. Nature 454: 455-462, 2008

40. Hotamisligil GS: Endoplasmic reticulum stress and the inflammatory basis of metabolic disease. Cell 140: 900-917, 2010.

41. Sabokbar A, Itonaga I, Sun SG, Kudo O and Athanasou NA Arthroplasty membrane-derived fibroblasts directly induce osteoclast formation and osteolysis in aseptic loosening. J Orthop Res 23: 511-519, 2005. 